\title{
Contributions à la modélisation simplifiée de la mécanique des contacts roulants
}

\author{
Lud $_{\text {Chevalier }}^{1, a}$, Sylvain Clouper ${ }^{2}$ et Anissa Eddhahak-Ouni ${ }^{1}$ \\ 1 Laboratoire de Mécanique, Université de Marne La Vallée, 5 boulevard Descartes, 77454 Marne La Vallée Cedex 2, France \\ 2 LMT-Cachan, École Normale Supérieure de Cachan, 61 avenue du Président Wilson, 94230 Cachan Cedex, France
}

Reçu le 11 novembre 2004, accepté le 21 novembre 2005

\begin{abstract}
Résumé - La modélisation de l'usure superficielle nécessite la mise en place d'un outil numérique de calcul des puissances dissipées lors du roulement sous charge de galets sur des pistes de came [1]. Compte tenu des dispersions relatives aux défauts géométriques et aux paramètres mal connus comme le coefficient de frottement par exemple, l'étude s'oriente vers une approche probabiliste [2] où le nombre de cas simulés devient très grand et hors d'atteinte en des temps de calcul CPU raisonnables par une approche exacte. L'utilisation d'une approche simplifiée est alors nécessaire pour le calcul de la puissance dissipée dans le cas non hertzien généré par l'usure. Pour quantifier la précision (ou l'incertitude) d'une telle approche, nous présentons et discutons les choix qui ont été réalisés; en particulier, l'approche simplifiée proposée par Kalker [3] est comparée avec les solutions de l'approche exacte, puis nous proposons des améliorations aux approches semi-hertziennes déjà utilisées dans [4] ou [5].
\end{abstract}

Mots clés : Mécanique du contact / roulement stationnaire / puissance dissipée / solides hertziens / quasi identité / méthode des éléments diffus

\begin{abstract}
Contribution to the simplified modelling of rolling contact mechanic. Numerical simulation of superficial wear requires the implementation of computational tools for calculation of dissipated power during loaded contacts of roller on track cam [1]. Uncertainties due to geometrical defects or to not well known parameters such as the friction ratio for example, yield to a probabilistic approach [2] of the contact problem. Number of simulated cases becomes very important and cannot reasonably be treated by the exact approach which leads to long CPU time. A simplified approach was chosen to calculate the dissipated power for non-hertzian case generated by the wear. To quantify the precision of this approach, we present and discuss the choices which had been made. In particular, the simplified approach proposed by Kalker [3] is compared with the solutions of the exact approach, then we propose improvement of semi-hertzian approaches which are presented in [4] or [5].
\end{abstract}

Key words: Contact mechanic / steady state rolling / dissipated power / hertzian solids / quasiidentity / diffuse elements method

\section{Introduction}

Les bureaux d'études ont besoin d'outils de calculs prédictifs, fiables et rapides pour le dimensionnement de leurs composants et notamment pour la prédiction de l'usure rencontrée dans les contacts roulants. La simulation de l'usure est un point clef de la prévision de la durée de vie des systèmes mécaniques.

La modélisation de cette usure superficielle passe par l'identification d'une loi d'usure, dérivée des travaux d'Archard [6], qui est effectuée sur un banc d'essai reproduisant le comportement à l'usure d'un galet roulant

\footnotetext{
a Auteur correspondant : Luc.Chevalier@univ-mlv.fr
}

sur une came. Ce banc industriel décrit dans [1], présente un nombre de paramètres mal maîtrisés et une approche probabiliste doit être mise en place pour envisager l'identification de la loi d'usure.

Devant le grand nombre de tirages à réaliser avec une telle approche, une modélisation par éléments finis ou par la méthode des éléments frontières du système d'équations à résoudre est tout à fait hors de portée et des méthodes de calculs simplifiées doivent être développées.

Tout d'abord, cet article présente la résolution du problème de contact entre deux solides élastiques décrite par Johnson [7] et Kalker [8], puis les solutions approchées utilisées sont présentées ainsi que des comparaisons aux 
solutions exactes dans un cas purement hertzien. Enfin, la troisième partie propose d'étendre les approximations aux cas non hertziens en rajoutant un terme de diffusion entre les bandes de contact pour « coller» au mieux à l'approche exacte.

\section{Résolution du problème de contact}

Afin de discuter la précision des approches simplifiées utilisées pour la simulation du contact roulant, nous allons brièvement rappeler les équations à résoudre dans un problème de contact entre deux solides élastiques $\mathbf{1}$ et 2. Ce problème, mis en équations depuis longtemps par Boussinesq [9] et Cerruti [10] s'appuie sur la connaissance des composantes du champ de déplacement $u_{b}, v_{b}$ et $w_{b}$ sous l'effet d'une charge ponctuelle de composantes $X_{b}$, $Y_{b}$ et $Z_{b}$ appliquée en un point de l'aire de contact. La position de ce point est définie par les coordonnées $\left(x^{\prime}, y^{\prime}\right)$ dans le repère où $\mathrm{Z}$ est la normale au plan tangent du contact.

Ainsi en négligeant la contribution des forces volumiques le problème se met sous la forme donnée par l'équation (1) où $u_{b}, v_{b}$ et $w_{b}$ sont des fonctions connues linéaires en $X_{b}, Y_{b}, Z_{b}$ et où $\tau_{x}$ et $\tau_{y}$ sont les contraintes tangentielles suivant la direction $x$ et suivant la direction $y$.

$$
\begin{array}{r}
\iint_{\text {contact }}\left(\tau_{x}\left(x^{\prime}, y^{\prime}\right) u_{b}+\tau_{y}\left(x^{\prime}, y^{\prime}\right) v_{b}+p\left(x^{\prime}, y^{\prime}\right) w_{b}\right) \mathrm{d} x^{\prime} \mathrm{d} y^{\prime}= \\
u(x, y) X_{b}+v(x, y) Y_{b}+w(x, y) Z_{b}
\end{array}
$$

Il convient donc de résoudre l'équation (1) pour chacun des solides avec de plus les conditions d'interface sur la zone de contact. Dans cette zone les forces surfaciques s'opposent et les déplacements normaux sont nuls. Par ailleurs, s'il y a du frottement entre les deux solides il convient d'introduire des lois de frottement. Dans la suite, nous utiliserons la loi de Coulomb avec $\mu$ le coefficient de frottement.

\subsection{Chargement normal}

Dans le cas où la charge appliquée est uniquement portée par $Z$ et où le frottement est négligé (c'est-àdire $\tau_{x}=\tau_{y}=0$ ), l'équation (1) permet le calcul du déplacement $w(x, y)$ normal à l'aire de contact provenant de la déformation par l'équation (2) :

$$
w(x, y)=\frac{1-v}{2 \pi G} \iint_{\text {contact }}\left\{\frac{p\left(x^{\prime}, y^{\prime}\right)}{\sqrt{\left(x-x^{\prime}\right)^{2}+\left(y-y^{\prime}\right)^{2}}}\right\} \mathrm{d} x^{\prime} \mathrm{d} y^{\prime}
$$

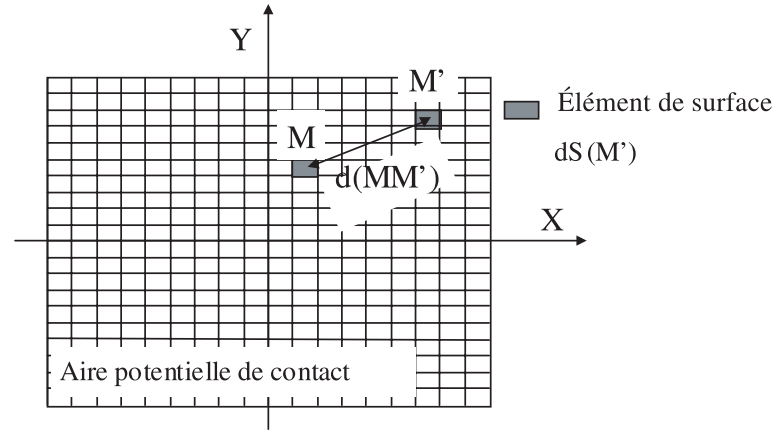

Fig. 1. Découpage de l'aire potentielle de contact.

Dans le cas d'un contact entre deux solides $\mathbf{1}$ (galet) et $\mathbf{2}$ (rondin) avec l'axe $Z$ dirigé de $\mathbf{2}$ vers $\mathbf{1}$, la différence de déplacement due à la déformation est donc donnée par :

$$
\begin{aligned}
w(x, y) & =w_{1}(x, y)-w_{2}(x, y) \\
& =\frac{1-v}{\pi G} \iint_{\text {contact }}\left\{\frac{p\left(x^{\prime}, y^{\prime}\right)}{\sqrt{\left(x-x^{\prime}\right)^{2}+\left(y-y^{\prime}\right)^{2}}}\right\} \mathrm{d} x^{\prime} \mathrm{d} y^{\prime} \\
\frac{1}{G} & =\frac{1}{2 G_{1}}+\frac{1}{2 G_{2}} \text { et } \frac{v}{G}=\frac{v_{1}}{2 G_{1}}+\frac{v_{2}}{2 G_{2}}
\end{aligned}
$$

Hertz [11] propose une solution analytique de l'équation (3) dans le cas où les courbures sont constantes au niveau du contact. La zone de contact est alors elliptique (dimensions $a$ et $b$ ) et la répartition de pression est ellipsoïdale. $P_{0}$ est donnée par l'équation (4). $a$ et $b$ sont déterminés à partir de coefficients $m$ et $n$ définis par des intégrales et généralement tabulés.

$$
P_{0}=\frac{3 F}{2 \pi a b}
$$

Lorsque les courbures des corps en contact ne sont pas constantes, la recherche du déplacement $w(x, y)$ et de la pression de contact $p(x, y)$ se fait en résolvant l'équation (5).

$$
\begin{aligned}
e(x, y) & =h(x, y)-\delta \\
+ & \frac{1-v}{\pi G} \iint_{\text {contact }}\left\{\frac{p\left(x^{\prime}, y^{\prime}\right)}{\sqrt{\left(x-x^{\prime}\right)^{2}+\left(y-y^{\prime}\right)^{2}}}\right\} \mathrm{d} x^{\prime} \mathrm{d} y^{\prime}
\end{aligned}
$$

L'écart $e$ entre les deux solides après déformation est donné par l'écart $h$ avant déformation, le rapprochement global $\delta$ loin de la zone de contact est une intégrale qui dépend de la pression $p$. Outre $e$ et $p$ qui sont inconnus, le premier à l'extérieur et le second à l'intérieur de la zone de contact, la zone de contact elle-même n'est pas connue.

La résolution du problème est numérique : l'aire potentielle de contact est découpée en $N$ éléments (Fig. 1). Sur chaque élément la pression inconnue est constante et l'intégrale est donc remplacée par une somme sur tous les éléments. Une méthode itérative permet de déterminer $e_{M}$ et $p_{M}$ de proche en proche par la démarche suivante : pour une valeur imposée de $\delta$, on initie les variables en considérant que tous les éléments sont 


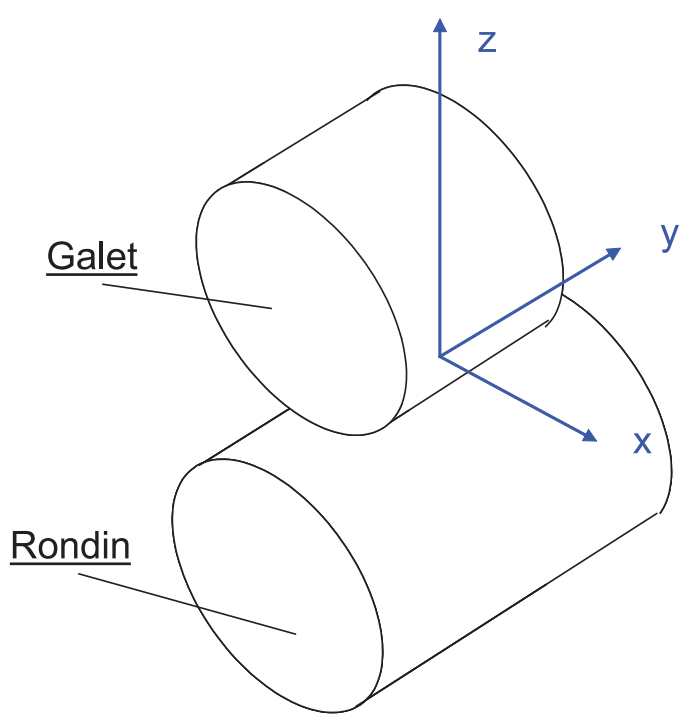

Fig. 2. Repérage des corps en contact.

extérieurs au contact, la pression est donc nulle partout et l'équation (5) permet l'estimation des $e_{M}$ initiaux.

Tous les éléments pour lesquels l'écart $e_{M}$ est négatif sont déclarés appartenant à l'aire de contact et leur écart est mis à 0 . Soit $P$ le nombre d'éléments qui changent ainsi d'état, on résout le nouveau système de $P$ équations ainsi obtenu et on détermine les $P$ valeurs de pression $p_{M}$ inconnues.

Tous les éléments pour lesquels la pression $p_{M}$ est négative sont déclarés ne faisant pas partie du contact et leur pression est mise à 0 . On recalcule les écarts $e_{M}$ avec les nouvelles valeurs de pression.

On itère ainsi entre le point $\mathbf{1}$ et le point $\mathbf{2}$ jusqu'à ce que toutes les pressions soient positives ou nulles et tous les écarts soient positifs ou nuls. Ceci permet de délimiter la zone de contact dans l'aire potentielle. La méthode est d'autant plus précise que le découpage de l'aire est fin mais conduit à des temps de calcul assez importants.

La méthode est illustrée (Fig. 2) sur le cas du contact normal entre un galet ( $\mathrm{g}$ ) et un rondin-éprouvette (e). Les deux solides sont de révolution en acier de caractéristiques géométriques : $R_{x \mathrm{~g}}=20 \mathrm{~mm}, R_{y \mathrm{~g}}=500 \mathrm{~mm}, R_{x \mathrm{e}}=$ $25 \mathrm{~mm}$ et $R_{y \text { e }}$ qui tend vers l'infini. Les caractéristiques élastiques du matériau valent : module d'Young $E=$ $2,1 \times 10^{11} \mathrm{~Pa}$, coefficient de Poisson $v=0,28$ et la charge appliquée est $F=1500 \mathrm{~N}$. Les courbures constantes et les relations de Hertz donnent la solution $a=0,23 \mathrm{~mm}$ et $b=2,63 \mathrm{~mm}$.

Numériquement, l'allure plus ou moins lisse de la pression de contact dépend du nombre de points servant à la description du profil du galet dans les différents plans principaux $(y O z)$ et $(x O z)$. La discrétisation adoptée pour le découpage de l'aire de contact influence elle aussi la finesse des résultats. Sur la figure 3 on observe les effets de ces deux discrétisations sur la répartition de pression et la forme de l'ellipse de contact. Pour un découpage assez fin $(100 \times 50)$ et une discrétisation des profils par plus de 40 points, les erreurs de géométrie entre la méthode numérique et le calcul analytique ne dépasse pas $1 \%$. Notons que l'élancement est assez fort et que le découpage doit être plus fin suivant $X$ que suivant $Y$.

L'approche numérique devient indispensable lorsque les courbures ne sont pas constantes au voisinage du point de contact. Dans le cas du galet-rondin, lorsque le point de contact se rapproche des flancs du galet, il y a un raccordement entre deux courbures très différentes. C'est un cas assez sévère (Fig. 4) que nous appelons « galet en biais ». Pour comparer de manière un peu systématique avec l'approche simplifiée qui sera présentée plus loin, on étudie plusieurs valeurs du rayon de raccordement entre 1 et $500 \mathrm{~mm}$.

Les formes des zones de contact sont données sur la figure 5. Lorsque le rayon de raccordement est faible, l'élancement de la zone de contact, située en $y<0$, est plutôt dirigée suivant $X$ puisque le rayon du galet dans le plan $(x O z)$ est plus élevé $\left(R_{y g}=20 \mathrm{~mm}\right)$. L'élancement suivant $X$ diminue au fur et à mesure que le rayon de raccordement augmente pour redonner l'ellipse de Hertz pour $R_{1}=R_{2}$.

\subsection{Problème complet du contact roulant}

Dans le cas où le frottement n'est pas négligeable, des cisaillements apparaissent qui sont liés aux déplacements relatifs par l'équation (6) pour deux solides de caractéristiques élastiques identiques.

$$
\begin{aligned}
u(x, y)= & \frac{1}{\pi G} \iint_{\text {contact }}\left(\left\{\frac{1-v}{r}+\frac{v\left(x-x^{\prime}\right)^{2}}{r^{3}}\right\} \tau_{x}\left(x^{\prime}, y^{\prime}\right)\right. \\
& \left.+\frac{v\left(x-x^{\prime}\right)\left(y-y^{\prime}\right)}{r^{3}} \tau_{y}\left(x^{\prime}, y^{\prime}\right)\right) \mathrm{d} x^{\prime} \mathrm{d} y^{\prime} \\
v(x, y)= & \frac{1}{\pi G} \iint_{\text {contact }}\left(\frac{v\left(x-x^{\prime}\right)\left(y-y^{\prime}\right)}{r^{3}} \tau_{x}\left(x^{\prime}, y^{\prime}\right)\right. \\
& \left.+\left\{\frac{1-v}{r}+\frac{v\left(y-y^{\prime}\right)^{2}}{r^{3}}\right\} \tau_{y}\left(x^{\prime}, y^{\prime}\right)\right) \mathrm{d} x^{\prime} \mathrm{d} y^{\prime} \\
w(x, y)= & \frac{1-v}{\pi G} \iint_{\text {contact }} \frac{p\left(x^{\prime}, y^{\prime}\right)}{r} \mathrm{~d} x^{\prime} \mathrm{d} y^{\prime}
\end{aligned}
$$

Ces équations montrent un découplage du problème normal avec le problème tangent. Réciproquement, bien que la pression n'intervient pas explicitement dans les deux premières équations, les cisaillements $\tau_{x}$ et $\tau_{y}$ ne sont pas indépendants de la pression puisqu'ils sont liés par l'inégalité de la loi de frottement de Coulomb (Éq. (7)) où $\mu$ est le coefficient de frottement et $\tau$ le module du cisaillement.

$$
\tau=\mu p \quad \text { avec } \quad \tau=\sqrt{\tau_{x}^{2}+\tau_{y}^{2}}
$$

Dans le cas du roulement stationnaire, le système d'équations cinématiques du contact galet/rondin est 

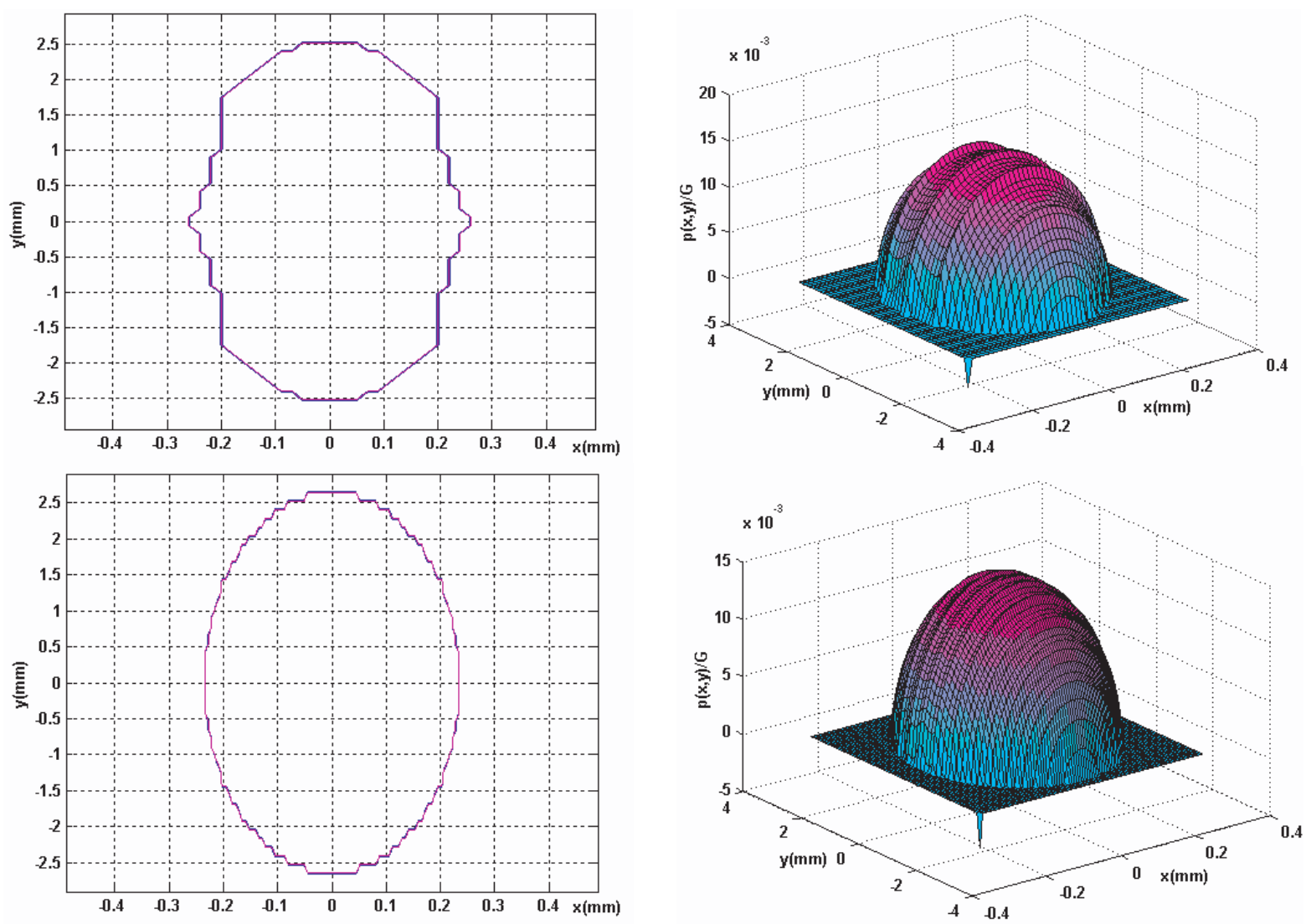

(a)

(b)

Fig. 3. (a) Influence de la discrétisation sur l'aire de contact pour un découpage de $50 \times 50$ (en haut) et $100 \times 50$ (en bas) de l'aire potentielle; (b) Influence de la description du profil du galet sur l'aspect de la répartition de pression : calculs avec respectivement 20 points (en haut) et 40 points (en bas).

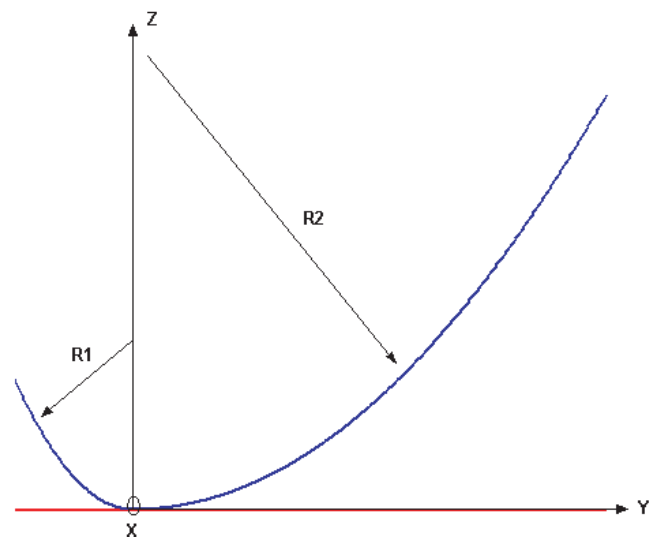

Fig. 4. Profil initial dans le plan $(y O z)$ d'un exemple non Hertzien : « galet en biais ».

donné par l'équation (8)

$$
\left\{\begin{array}{l}
\frac{w_{x}}{V}=\nu_{x}-\phi y-\frac{\partial u}{\partial x} \\
\frac{w_{y}}{V}=\nu_{y}+\phi x-\frac{\partial v}{\partial x}
\end{array}\right.
$$

où $w_{x}$ et $w_{y}$ désignent les composantes de la vitesse de glissement entre les deux corps, $V$ la vitesse de renouvellement de contact, $\nu_{x}, \nu_{y}$ et $\phi$ sont respectivement les pseudo glissements longitudinal, transversal et de spin, $u$ et $v$ sont les composantes du déplacement relatif entre les deux solides provenant des déformations présentées dans l'équation (6).

La démarche de résolution est itérative : on suppose un état initial pour lequel la zone d'adhérence est confondue avec la zone de contact. Le système (8) permet le calcul des déplacements $u$ et $v$. L'équation (6) permet de déterminer $\tau_{x}$ et $\tau_{y}$ par inversion. La condition de l'équation (7) permet ensuite de distinguer les points où le cisaillement sature (zone de glissement) et ceux pour lesquels il y a adhérence. Cette partition permet d'affiner la zone où $w_{x}=w_{y}=0$. On réitère la démarche jusqu'à stabilisation entre zones d'adhérence et de glissement.

La figure 6 montre cette partition dans le cas du contact galet-rondin à courbures constantes. Les données supplémentaires par rapport à l'exemple du contact normal sont : coefficient de frottement $\mu=0,1$, pseudo glissement longitudinal $\nu_{x}=10^{-3}$, pseudo glissement longitudinal $\nu_{y}=0$, pseudo glissement de spin $\phi=0$ et la vitesse de renouvellement du contact $V=2,6 \mathrm{~m} \cdot \mathrm{s}^{-1}$. La zone de contact est découpée en une zone d'adhérence 

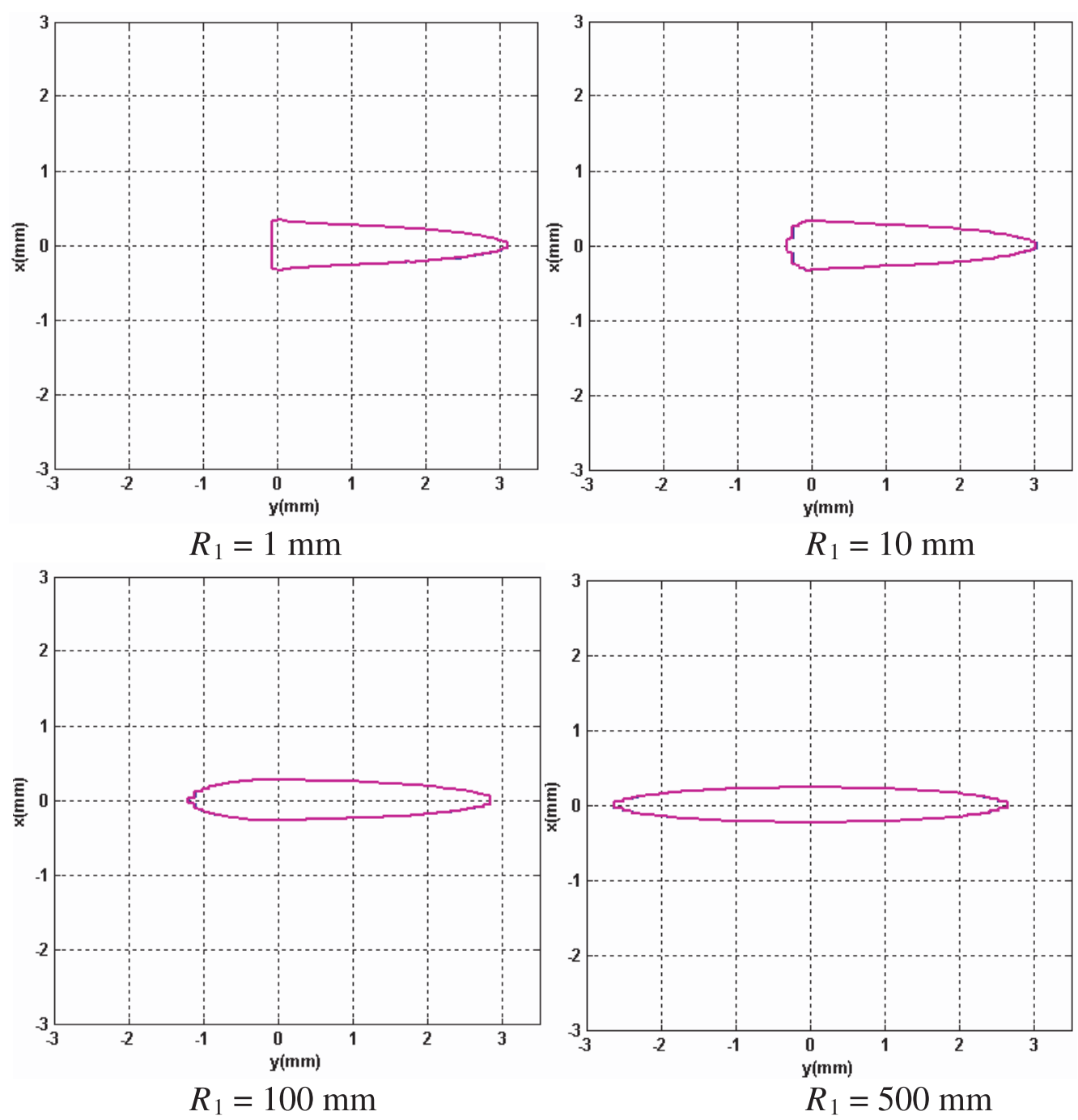

Fig. 5. Formes de la zone de contact données par l'approche exacte dans le cas où le rayon de bombé du galet vaut 500 mm.

et une zone de glissement. Les forces surfaciques tangentielles ont un aspect quasiment linéaire sur la zone d'adhérence puis une forme elliptique à la saturation. Les vitesses de glissement sont nulles sur la partie collante et augmentent linéairement dans la zone de glissement atteignant ainsi un maximum fini sur le bord de fuite de l'ellipse de contact.

Notons que le problème de roulement stationnaire décrit par les équations (6-8) trouve une solution analytique presque exacte lorsqu'on fait l'hypothèse d'adhérence totale (ce qui correspond à un frottement très fort ou à des pseudo glissements très faibles). Dans ce cas le système de l'équation (8) donne $u$ et $v$ et par identification avec les expressions de l'équation (6), on peut en déduire les charges tangentielles résultantes $T_{x}$ et $T_{y}$ ainsi que le moment résultant $M_{z}$ qui s'oppose au pivotement : c'est un résultat de la théorie linéaire de Kalker (TLK) développé dans [12].

Dans les deux parties suivantes, nous allons discuter les méthodes approchées présentées en comparant leurs performances aux résultats de l'approche exacte.

\section{Résolution approchée du contact roulant}

Dans cette partie, nous présentons la résolution simplifiée proposée par Kalker [12] et qui est à l'origine de l'algorithme FastSim. L'hypothèse simplificatrice de la mise en équations par FastSim est appelée « tapis de ressort ». Elle considère la proportionnalité entre les déplacements et les efforts de cisaillement de manière globale par les relations :

$$
\left\{\begin{array}{l}
u=L \tau_{x} \\
v=L \tau_{y}
\end{array}\right.
$$

où $L$ est la flexibilité de contact.

L'identification des résultantes $T_{x}$ et $T_{y}$ obtenues par résolution du système (9) et le système (8) avec celles de la théorie linéaire de Kalker n'est possible que si l'on considère trois coefficients de flexibilité distincts $L_{1}, L_{2}$ et $L_{3}$ associés respectivement aux pseudo glissements $\nu_{x}$, $\nu_{y}$ et $\phi$ qui sont de la forme :

$$
L_{1}=\frac{8 a}{3 C_{11} G}, \quad L_{2}=\frac{8 a}{3 C_{22} G}, \quad L_{3}=\frac{\pi a \sqrt{a / b}}{4 C_{23} G}
$$



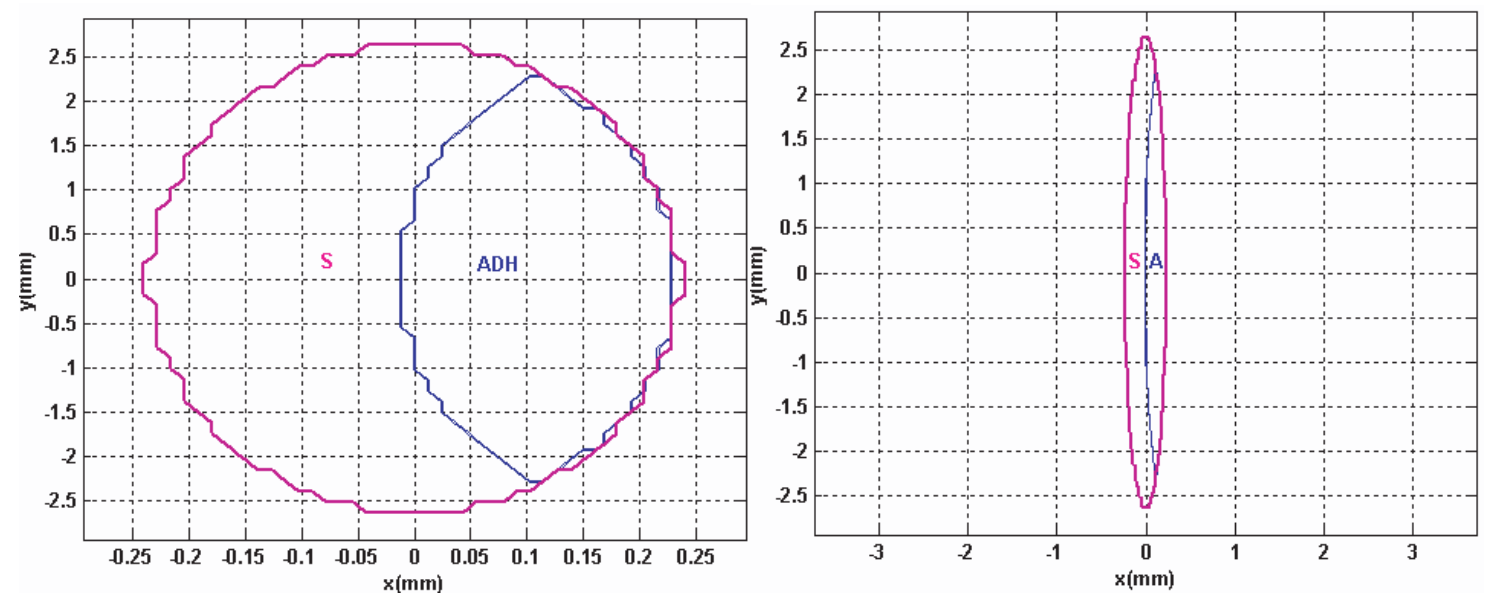

(a)

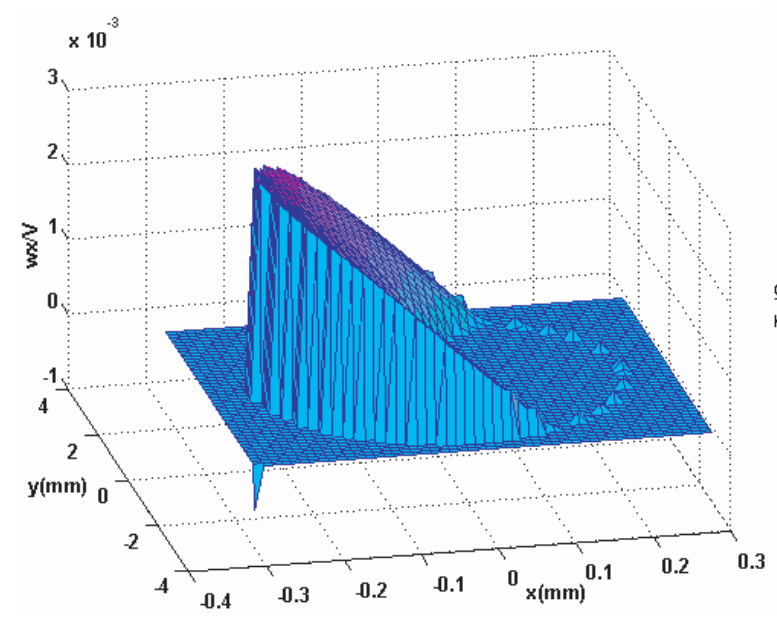

(b)

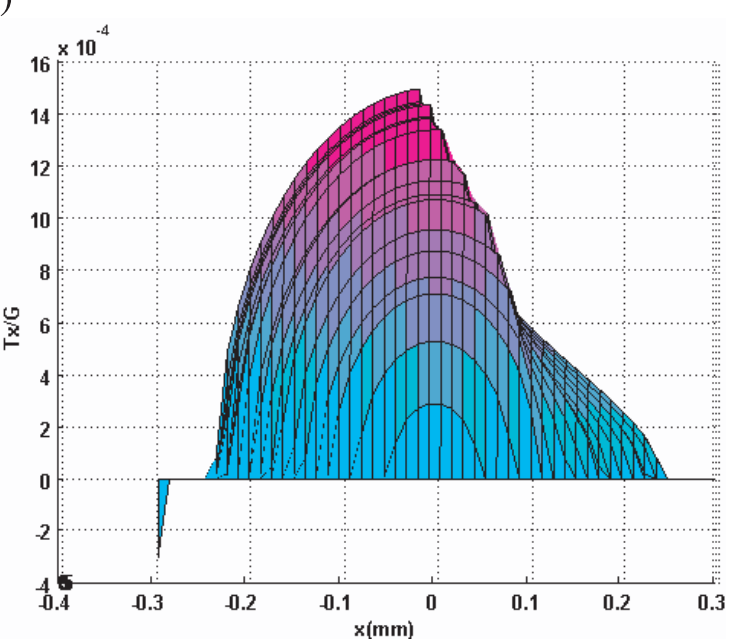

(c)

Fig. 6. Résultats de l'approche exacte dans le cas du roulement stationnaire : (a) Forme de la zone de contact (avec ou sans respect des proportions), (b) vitesse de glissement $w_{x}$, (c) densité surfacique de forces tangentielles au contact $\tau_{x}$.

Dans le cas où tous les pseudo glissements coexistent, Soua [13] propose une flexibilité moyenne par :

$$
L=\frac{L_{1}\left|v_{x}\right|+L_{2}\left|v_{y}\right|+L_{3}|\varphi \sqrt{a b}|}{\sqrt{v_{x}^{2}+v_{y}^{2}+\varphi^{2} a b}}
$$

En définitif, le système d'équations à résoudre par FastSim sera donc :

$$
\left\{\begin{array}{l}
\frac{w_{x}}{V L}=\frac{\nu_{x}}{L_{1}}-\frac{\phi y}{L_{3}}-\frac{\partial \tau_{x}}{\partial x} \\
\frac{w_{y}}{V L}=\frac{\nu_{y}}{L_{2}}+\frac{\phi x}{L_{3}}-\frac{\partial \tau_{y}}{\partial x}
\end{array}\right.
$$

Il faut noter que :

(i) La définition des $L_{i}$ fait apparaître des dimensions globales de l'ellipse de Hertz et la généralisation aux contacts non hertziens n'est pas évidente;

(ii) Les résultats de cette approche seront d'autant plus corrects que le frottement sera fort ou les pseudo glissements seront faibles; (iii) Kalker n'utilise pas le moment $M_{z}$ dans son identification des $L_{i}$, or ce dernier n'est pas compatible avec les expressions de $L_{1}, L_{2}$ et $L_{3}$. Il faut donc prévoir des écarts importants lorsqu'il y a beaucoup du spin.

Pour illustrer cette démarche de résolution, nous considérons à nouveau la géométrie de contact galet-rondin.

\subsection{Exemple d'illustration par FastSim : deux cas de répartition de pression}

Le problème normal étant découplé du problème tangent, on considère dans un premier temps que la répartition normale de pression est elliptique (Fig. 7, colonne de gauche).

L'aire de contact est composée de 2 zones disjointes : adhérente et glissante. La zone d'adhérence part du bord d'attaque $x=a_{i}$ et limitée par une valeur de $x_{S}$ qui est proportionnelle à la largeur $2 a_{i}$ de la bande $y_{i}$ considérée. Au-delà de cette zone, les cisaillements saturent suivant la loi de frottement de Coulomb et il y a glissement jusqu'au 
Contraintes tangentielles $\tau_{\mathrm{x}}$ en MPa

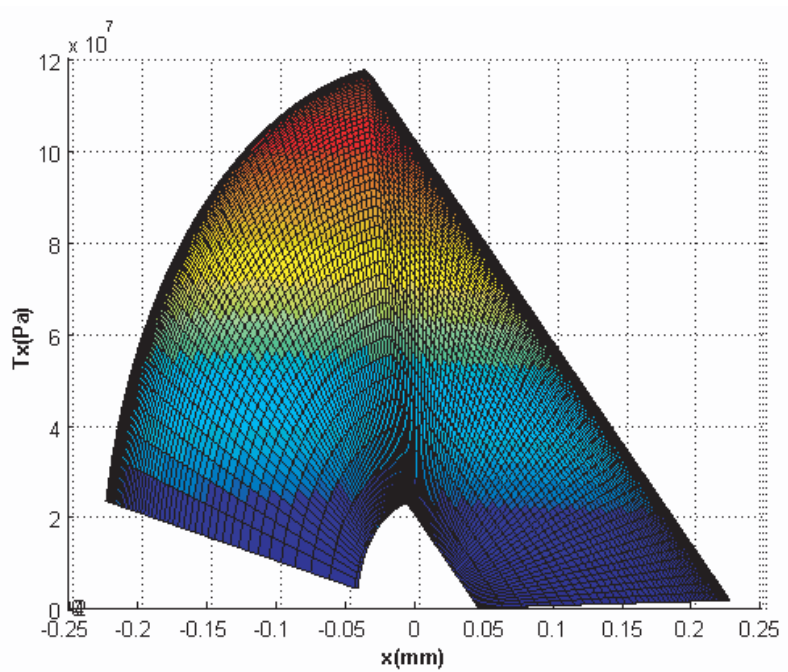

Vitesse de glissement $w_{\mathrm{x}}$ en $\mathrm{m} / \mathrm{s}$

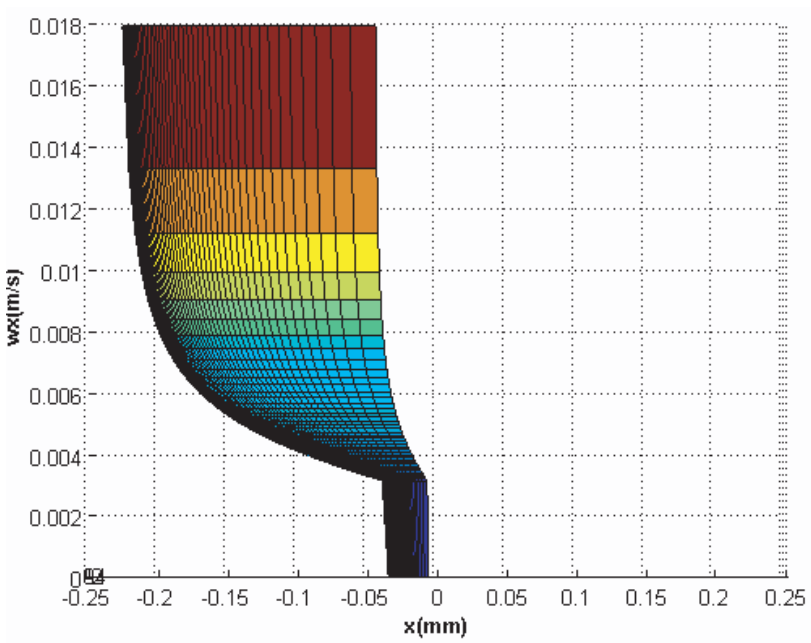

Puissance surfacique en $\mathrm{w} / \mathrm{m}^{2}$

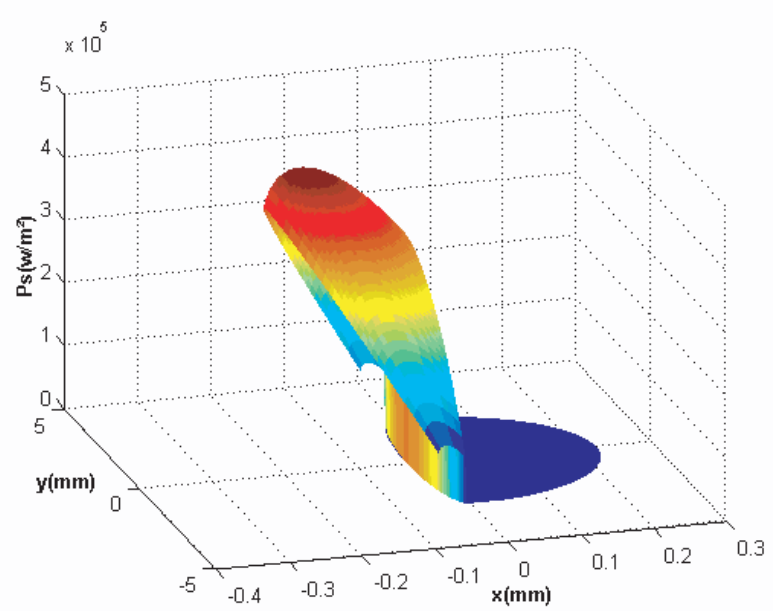

Contraintes tangentielles $\tau_{\mathrm{x}}$ en MPa

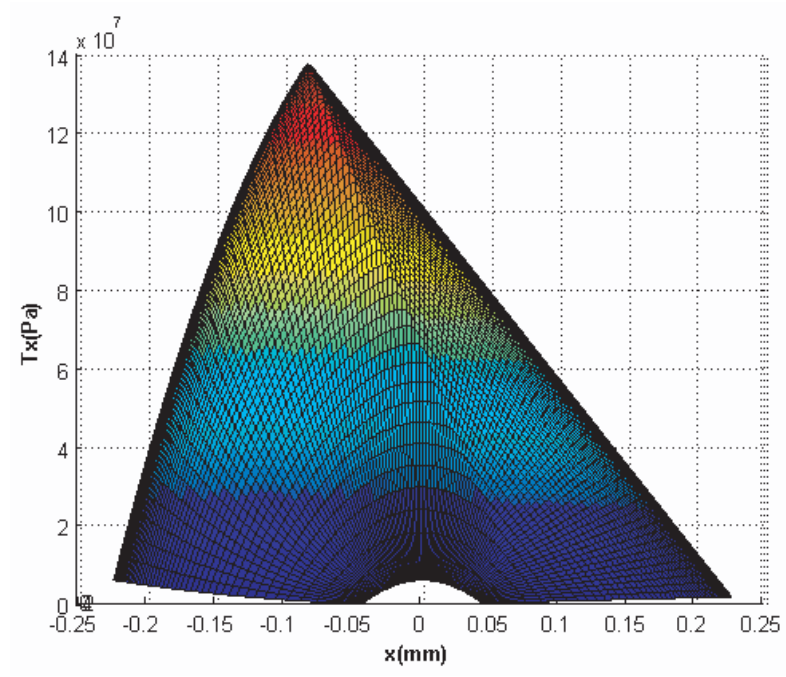

Vitesse de glissement $w_{\mathrm{x}}$ en $\mathrm{m} / \mathrm{s}$

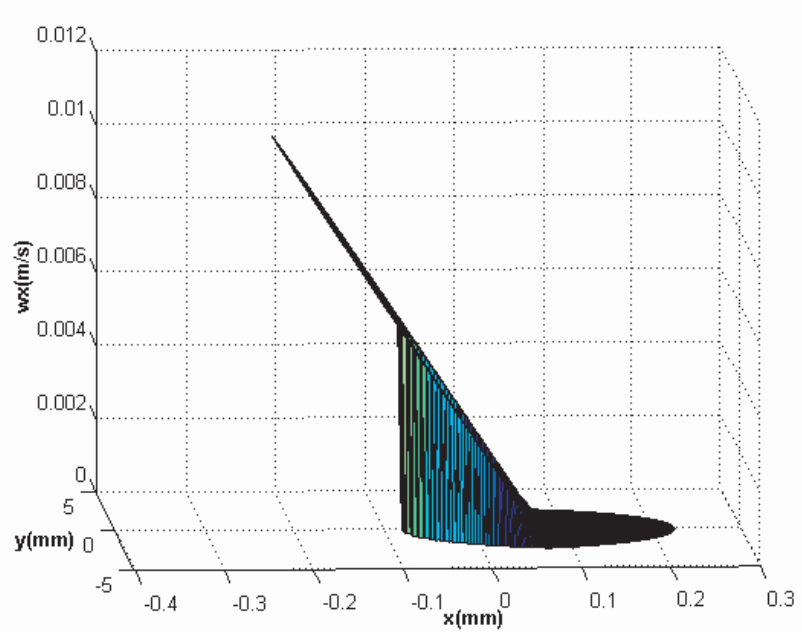

Puissance surfacique en $\mathrm{w} / \mathrm{m}^{2}$

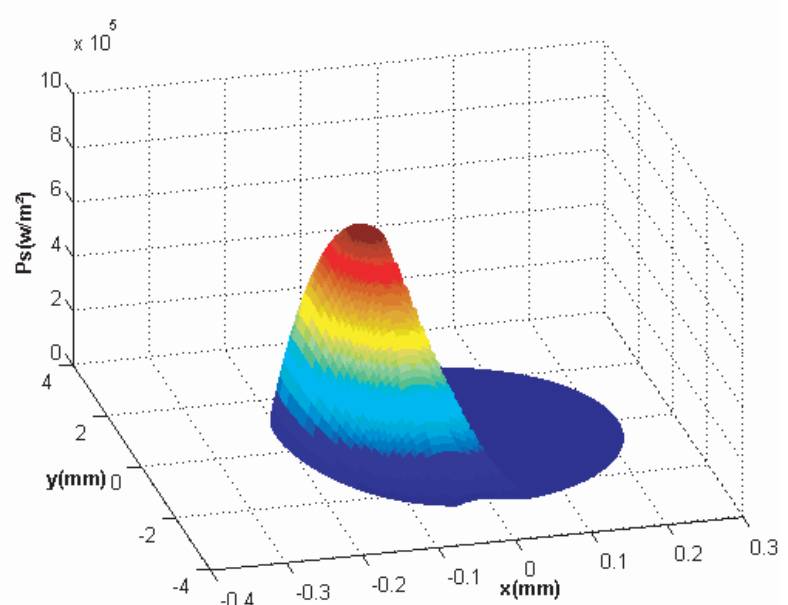

Fig. 7. Résultats de l'approche simplifiée dans le cas du roulement stationnaire. Colonne de gauche : répartition elliptique de pression; colonne de droite : répartition parabolique de pression. 
Tableau 1. Chiffrage des erreurs entre les trois approches.

\begin{tabular}{cccc} 
& Puissance surfacique $\max \left(10^{5} \mathrm{~W} \cdot \mathrm{m}^{-2}\right)$ & Puissance linéique max, $\left(\mathrm{W} \cdot \mathrm{m}^{-1}\right)$ & Puissance totale $(\mathrm{W})$ \\
\cline { 2 - 4 } Contact & 4,71 & 79,75 & 0,297 \\
FastSim (elliptique) & 4,81 & 85,51 & 0,29 \\
Erreur (\%) & $\mathbf{2 , 1 2}$ & $\mathbf{7 , 2}$ & $\mathbf{0 , 6 7}$ \\
FastSim (parabolique) & 8,01 & 86,77 & 0,28 \\
Erreur (\%) & $\mathbf{7 0}$ & $\mathbf{8 , 8}$ & $\mathbf{3 , 3}$ \\
\hline
\end{tabular}

bord de fuite $x=-a_{i}$. Dans cette zone, le système (12) donne la vitesse de glissement. On notera l'allure asymptotique au voisinage du bord de fuite. Néanmoins, la puissance surfacique dissipée au contact reste finie sur toute la zone de contact.

Comme alternative à cette singularité, Kalker propose une répartition parabolique de pression ne conduisant pas à une vitesse infinie (Fig. 7, colonne droite).

Dans le cas de la répartition parabolique, certaines bandes de l'ellipse sont totalement glissantes. Ces bandes, qui se situent aux extrémités de l'ellipse, ne présentent pas de zone d'adhérence. Ceci est en contradiction avec l'hypothèse d'un contact collant sur le bord d'attaque. La vitesse de glissement présente une discontinuité lors de la transition entre la zone collante et la zone glissante (comme dans le cas elliptique), mais on peut observer qu'elle reste finie et d'allure linéaire.

\subsection{Comparaison avec l'approche exacte et validation du chargement dans FastSim}

Le découpage de la zone de contact diffère entre l'approche exacte et les deux répartitions de pression de l'approche simplifiée. La saturation n'est pas atteinte au même endroit pour les trois modèles, ce qui résulte en une zone d'adhérence plus petite par la théorie exacte que celle par FastSim. Bien que la répartition parabolique donne une meilleure allure de saturation, le cas elliptique fournit des zones de découpages d'aires plus voisines de l'approche exacte comme l'illustre la figure 8.

En terme de puissance dissipée au contact, nous obtenons des valeurs finies par les trois modèles. Le tableau 1 chiffre le maximum des puissances surfacique et linéique et quantifie l'écart par rapport au modèle exact.

Cette comparaison milite pour le chargement elliptique dans l'approche simplifiée. Hormis l'allure non physique de la vitesse de glissement, le cas elliptique donne des résultats plus proches du calcul exact. L'allure de la vitesse de glissement est qualitativement satisfaisante en parabolique, quantitativement, l'écart reste très important.

On définit la puissance linéique comme la somme des puissances surfaciques le long d'une ligne $y=$ constante et la puissance totale comme la somme de ces puissances sur toute l'ellipse de contact. Une fois de plus, la comparaison montre que le cas parabolique dissipe légèrement plus que le cas elliptique comme montré dans la figure 9 . En terme de puissance linéique, les deux cas de chargement surestiment cette variable par rapport à l'approche exacte.

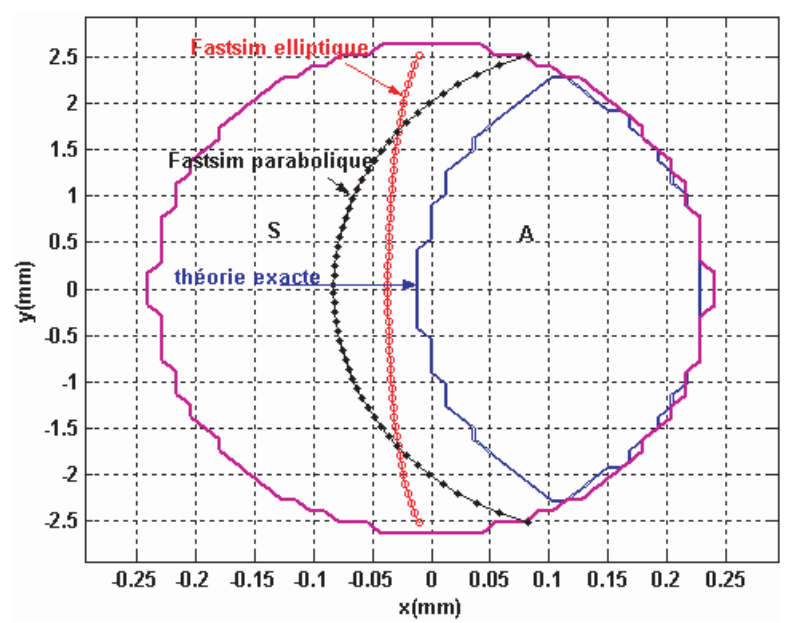

Fig. 8. Comparaison de la partition glissement $(S)$ /adhérence (A) de l'aire de contact par les trois approches. L'approche simplifiée surestime la zone d'adhérence quelle que soit la répartition de pression retenue. L'allure de la zone d'adhérence est meilleure dans le cas parabolique.

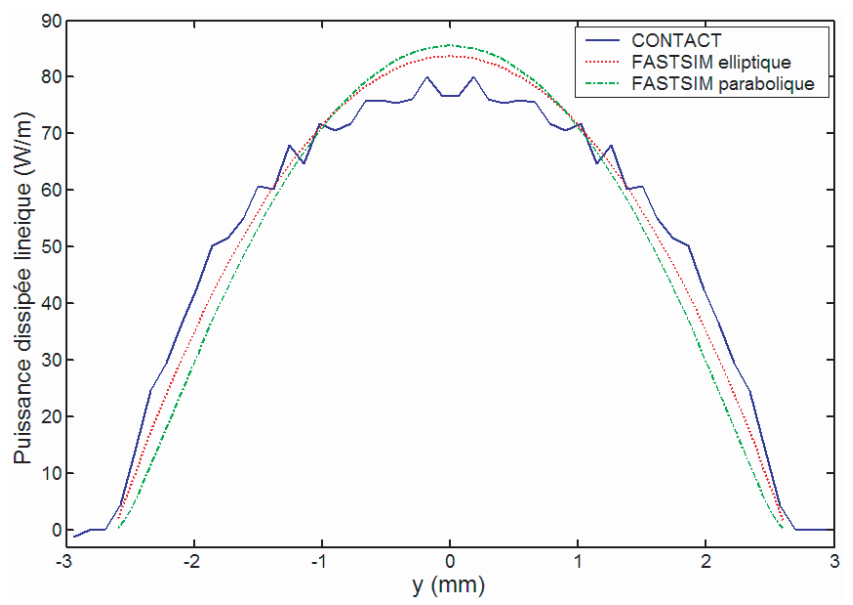

Fig. 9. Distribution de la puissance dissipée linéique par l'approche exacte (CONTACT) (calculée avec 10 points pour la description du profil et une discrétisation de $50 \times 50$ ) et par l'approche simplifiée dans le cas elliptique (FASTSIM elliptique) et dans le cas parabolique (FASTSIM parabolique).

Toutes ces constatations nous laissent privilégier le chargement elliptique dans le modèle simplifié FastSim. Dans la suite, seul ce type de chargement sera considéré. 


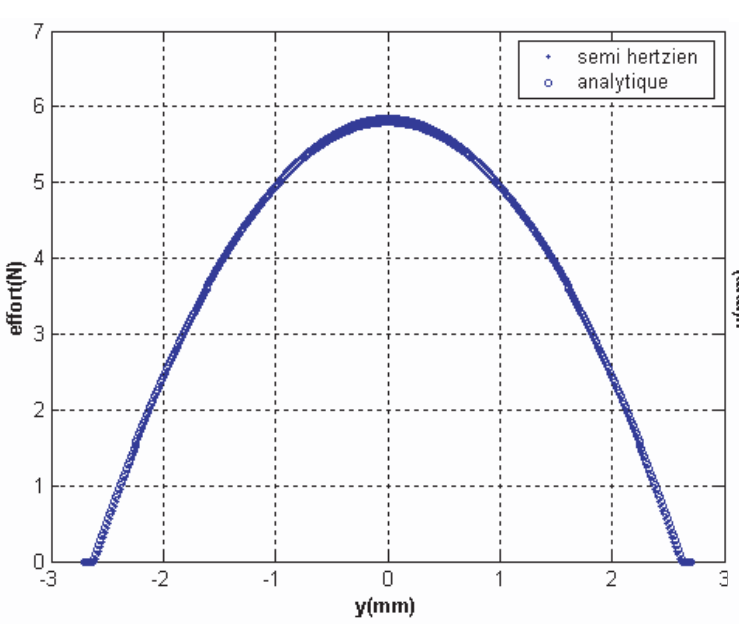

(a)

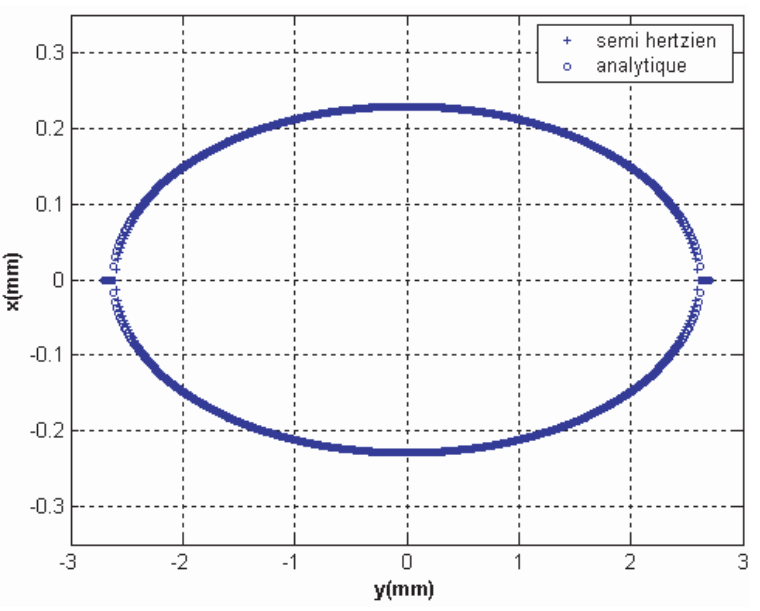

(b)

Fig. 10. Comparaison entre l'approche semi-hertzienne et la solution de Hertz sur un contact à courbures constantes : (a) distribution des efforts par bande, (b) forme de la zone de contact.

\section{Approche semi-hertzienne}

\subsection{Principe de l'approche semi-hertzienne}

FastSim est un outil de calcul rapide mais limité aux corps hertziens. Dès lors que les courbures ne sont plus constantes (après usure par exemple), il faut être capable de déterminer la zone de contact avant le calcul des efforts tangentiels. À partir des travaux de Kik et Piotrowski [14], divers auteurs proposent une approche dite semi-hertzienne analogue à Telliskivi [15]. L'idée consiste à estimer la zone de contact en se basant sur la pénétration virtuelle des corps à l'état non déformé. L'intersection des surfaces des corps limite une forme de contact. Cependant dans le cas hertzien, cette approche donne une forme elliptique ayant un élancement différent que celui de Hertz. C'est ainsi qu'une correction est mise en place consistant à modifier la courbure initiale $A$.

Dans le cas semi-hertzien, les deux corps sont de révolution mais de profils quelconques et le principe de correction doit se faire bande par bande.

Dans ce qui suit, nous présentons brièvement la démarche de résolution du problème semi-hertzien :

- On choisit une interpénétration $h_{0}$ et on délimite la largeur de la zone intersection entre les deux profils non déformés.

- On découpe cette largeur en $M M_{y}$ bandes de largeur $\Delta y$.

- Sur chaque bande autour de l'abscisse $y=y_{i}$, on calcule les courbures $A_{i}$ et $B_{i}$ et on effectue la correction $A_{c i}$ de la courbure $A_{i}$ grâce aux relations :

$$
\begin{gathered}
A_{c i}=\beta^{2} \cdot \lambda_{i}^{2 \alpha-1} A_{i} \\
\text { où } \quad \lambda_{i}=\frac{A_{i}}{B_{i}}
\end{gathered}
$$

et $\beta$ un coefficient figurant dans l'interpolation en loi puissance de l'élancement hertzien $k_{i}$ (qui tient compte de la déformation) et l'élancement dû à l'interpénétration virtuelle des deux solides donné par :

$$
k_{i}=\beta \cdot \lambda_{i}^{\alpha}
$$

- On calcule sur chaque bande le rapprochement local $h$ et la différence des pentes $\gamma_{i}$ par la relation :

$$
h_{i}=h_{0}-z_{g i}
$$

Cela permet de caractériser la géométrie locale de la zone de contact dans une bande d'abscisse $y=y_{i}$ :

$$
a_{i}=\sqrt{\frac{h_{i}}{A_{c i}}}
$$

- En considérant une répartition elliptique de chargement, on peut calculer la contribution en effort de chaque bande $\Delta N_{i}$ par la formule :

$$
\Delta N_{i}=\frac{E h_{i}}{2\left(1-v^{2}\right)} \cdot \frac{1+\lambda_{i}}{n_{i}^{3}} \cdot \Delta y
$$

On vérifie si la somme de ces charges sur toute l'aire de contact est égale à la charge normale globale $F$ : c'est le critère de convergence de la méthode.

- Nous augmentons ou réduisons la valeur de l'interpénétration en fonction du résultat choisi jusqu'à vérifier le critère :

$$
\sum_{i} \Delta N_{i}=F
$$

La pertinence de la méthode est tout d'abord testée sur le cas assez sévère (fort élancement) du contact galetrondin. Lorsque les courbures sont constantes, la méthode doit permettre de retrouver l'ellipse de Hertz.

Le résultat obtenu est évidemment excellent : les valeurs de $a$ et $b$ valent 2,8 et $0,24 \mathrm{~mm}$, soit un ratio $b / a$ de 11,48. Sur la figure 10, on représente : (a) la distribution 

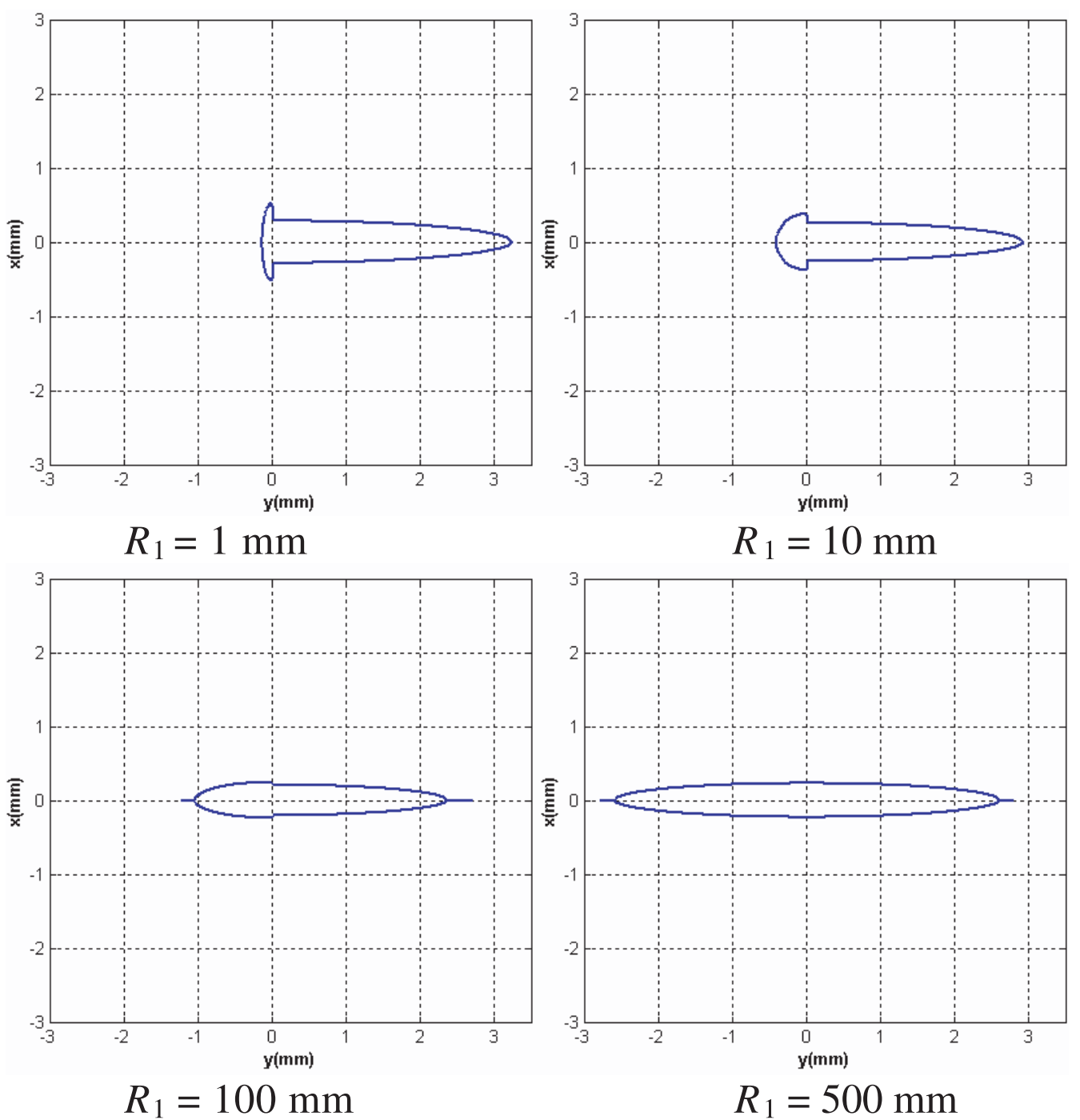

Fig. 11. Formes de la zone de contact données par l'approche semi-hertzienne pour des rayons de raccordement allant de 1 à $500 \mathrm{~mm}$ : avec l'accroissement de ces derniers, les pointes s'estompent peu à peu et on retrouve finalement l'ellipse de Hertz avec le bon élancement.

$\Delta N i$ obtenue en superposition de l'expression analytique et (b) les dimensions $a_{i}$ de l'ellipse de contact en superposition de l'ellipse de Hertz (théorique).

La figure 11 présente la forme de la zone de contact pour des rayons de raccordement variables dans le cas du galet en biais décrit à la section 1.2.3. Lorsque le rayon de raccordement est égal au rayon initial du galet $(500 \mathrm{~mm})$, nous retrouvons l'ellipse de Hertz. Pour des rayons de raccordement plus faibles, nous constatons l'apparition de pointes au niveau de la zone de transition entre les deux courbures. Ces pointes sont d'autant plus grandes que le rayon de raccordement est petit. Elles sont dues à la variation brutale de la courbure.

Comparant les résultats du modèle semi-hertzien aux résultats de l'approche exacte présentés à la section 1.2.3, nous constatons que ces pointes sont plus marquées avec l'approche semi-hertzienne. Néanmoins, les dimensions de l'aire de contact sont très voisines et varient de la même manière que pour l'approche exacte.
L'origine de ces pointes provient du découplage entre les caractéristiques élastiques des bandes. Nous proposons d'atténuer l'effet de ce découplage par une diffusion d'information entre les bandes.

\subsection{Diffusion entre les bandes}

Le principe de résolution de la méthode est toujours conservé. Néanmoins, pour contourner les effets du découplage nous allons régulariser l'allure du profil du galet dans le plan $(y O z)$. Les méthodes de lissage sont assez nombreuses et nous adopterons une diffusion par la méthode des éléments diffus [16] et [17].

La méthode des éléments diffus est utilisée dans la discrétisation des problèmes des milieux continus sans maillage. Elle consiste à trouver une approximation nodale d'une fonction définie par un nombre $N$ fini de points d'abscisse $y_{j}$. Elle donne ainsi accès, en ces points, aux $K$ 
premières dérivées ( $K$ ordre de la méthode) de la fonction initialement discrète. Appliquée à la courbure $B(y)$, cette approximation génère en un point une estimation du développement de Taylor à l'ordre $K$ de $B$ en $y$ de la forme

$$
B(y)=\sum_{i=0, K} p_{i}(y) \cdot \alpha_{i}^{*}(y)
$$

en choisissant le vecteur $\alpha^{*}(y)$ qui minimise l'écart quadratique :

$$
I_{y}(\alpha)=\sum_{j=1, N} w\left(y_{j}-y\right)\left[B\left(y_{j}\right)-p^{T}\left(y_{j}-y\right) \cdot \alpha(y)\right]^{2}
$$

où $N$ est le nombre de points de la discrétisation, $w$ est la fonction de pondération choisie positive, maximale à l'origine et rapidement décroissante en s'en éloignant, de la forme :

$$
w(\tilde{y})=\exp \left(-C \tilde{y}^{2}\right) \quad \text { avec }: \tilde{y}=\frac{y}{L_{y}}
$$

Il faut noter que le terme $\tilde{y}$ figurant dans l'équation (22) est sans dimension. $L_{y}$ est une dimension caractéristique du domaine d'étude de la fonction à régulariser. Le minimum de $I_{y}$ est atteint en $\alpha^{*}$ solution de :

$$
\begin{gathered}
A(y) \cdot \alpha(y)=b(y) \\
\text { avec : }\left\{\begin{array}{l}
A(y)=\sum w\left(y_{j}-y\right) p\left(y_{j}-y\right) p^{T}\left(y_{j}-y\right) \\
b(y)=\sum w\left(y_{j}-y\right) B\left(y_{j}\right) p\left(y_{j}-y\right)
\end{array}\right.
\end{gathered}
$$

Une fois résolu, le vecteur $\alpha$ permet d'obtenir la nouvelle fonction $B(y)$ par :

$$
\begin{aligned}
& B(y)=\alpha_{0}(y) \text { et } D^{m}(B(y))=m ! \alpha_{m}(y) \\
& \text { pour } m \text { variant de } 1 \text { à } K
\end{aligned}
$$

La figure 12 présente le résultat de la technique des éléments diffus d'une fonction bruitée telle que pourrait être un relevé de points sur un profil usé.

Les caractéristiques de la fonction de pondération $w$ génèrent un effet de lissage. L'accroissement du paramètre $C$ de l'équation (22) entraîne un élargissement de la notion de voisinage et par conséquent un lissage plus important. En revanche une diminution de ce paramètre fait rétrécir cette fenêtre, ce qui rend le lissage moins prononcé.

Cette observation nous permet d'adopter le meilleur paramètre $C$ pour réaliser le minimum d'erreur aux résultats obtenus par l'approche exacte. C'est ainsi que nous sommes amenés à rechercher le paramètre $C$ optimal en fonction de la plus ou moins brutale variation de courbure.

\subsection{Optimisation et comparaison avec la solution exacte}

Cette démarche consiste à calculer pour chaque rayon de raccordement le coefficient $C$ optimal qui réalise le
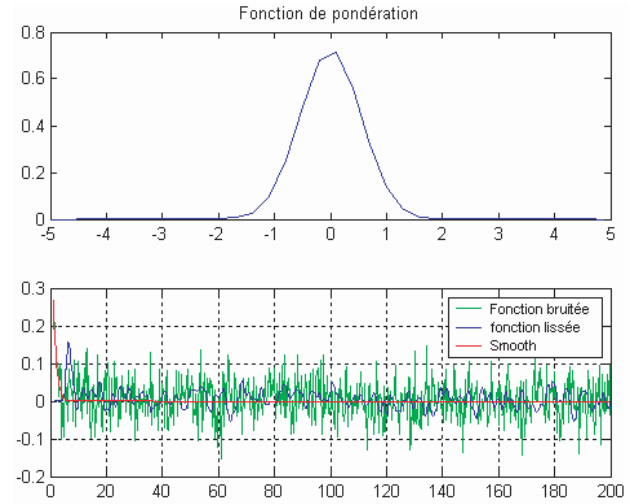

(a) $C=0,3$
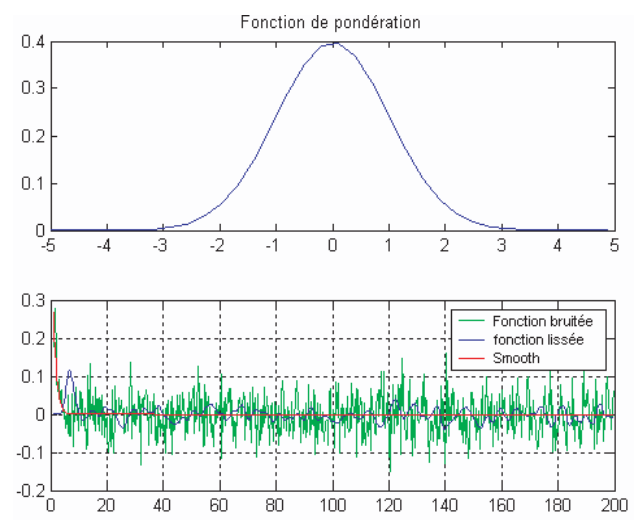

(b) $C=1$
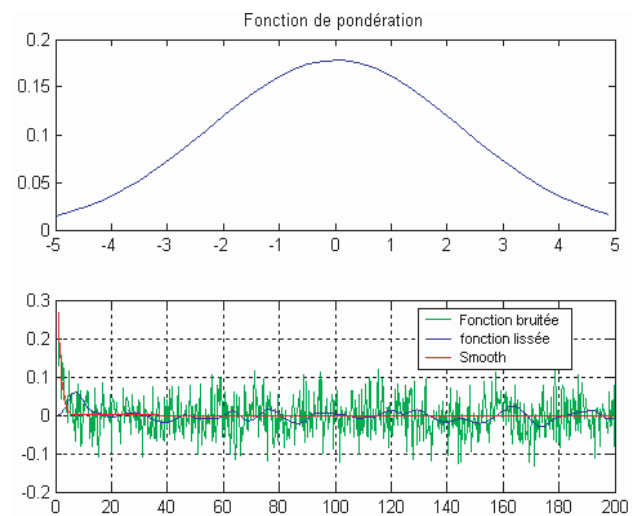

(c) $C=5$

Fig. 12. Influence des paramètres de la fonction de pondération sur la qualité de lissage : le cas (c) est plus lissé que les cas (a) et (b).

minimum de l'erreur défini comme suit :

$$
\operatorname{Erreur}(C)=\frac{\sum_{\text {bandes }} \sqrt{(\mathrm{d} S-\mathrm{d} s)^{2}}}{S}
$$

où : $\mathrm{d} S$ : aire de la bande $i$ donnée par la théorie exacte; $\mathrm{d} s$ : aire de la bande $i$ donnée par le modèle semi-hertzien 

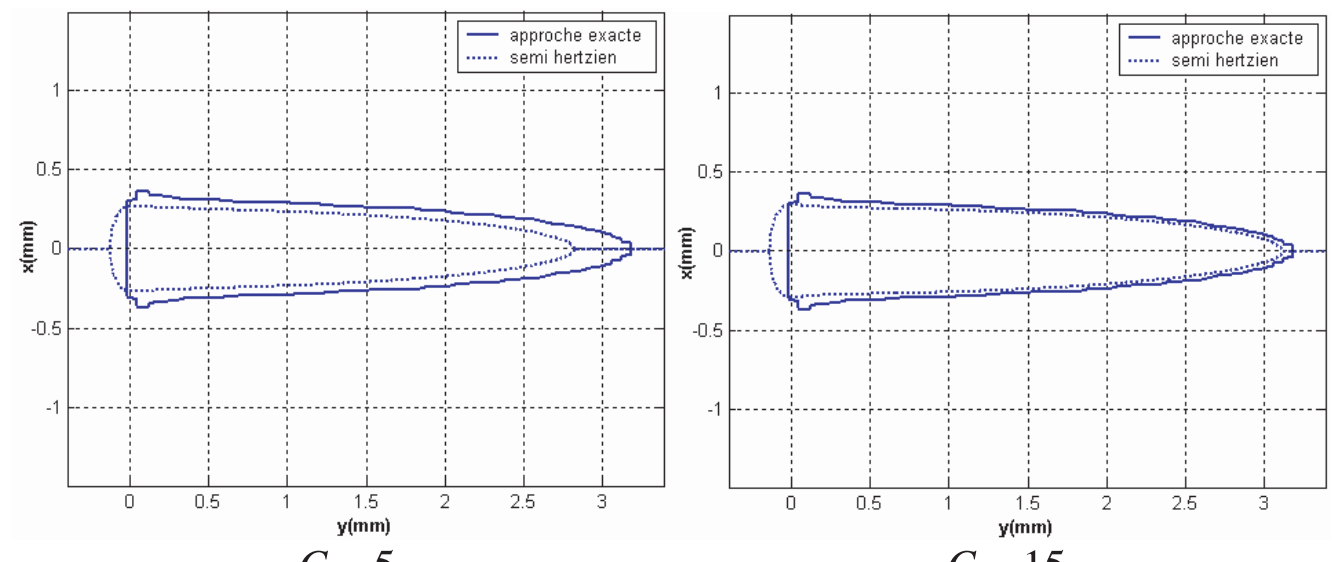

$C=5$
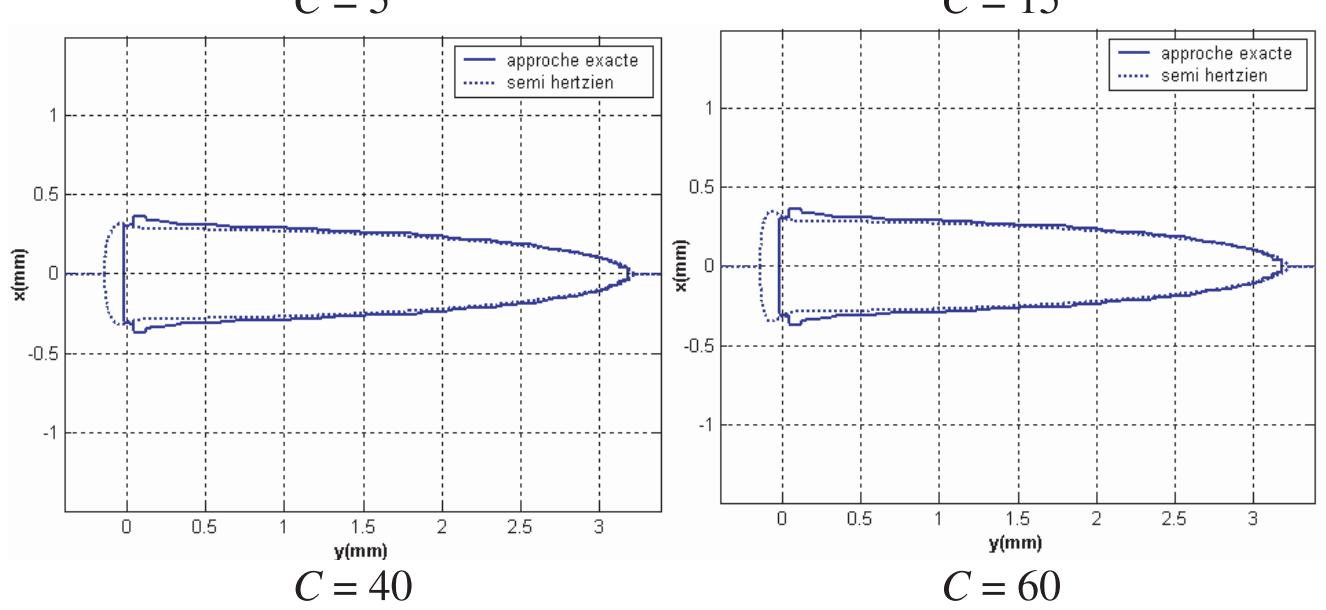

Fig. 13. Forme de la zone de contact en fonction du paramètre de diffusion $C$ dans le cas où le rapport $\frac{R_{1}}{R_{2}}=0,002$.

avec diffusion ; $S$ : aire de la zone de contact par la théorie exacte.

Cette démarche va nous permettre de trouver l'optimum des coefficients $C$ en comparant à chaque calcul l'aire de la zone de contact obtenue par la méthode semihertzienne avec l'aire exacte. Sur la figure 13, nous comparons quelques résultats de cet essai dans le cas extrême où le rapport $\frac{R_{1}}{R_{2}}=0,002$ pour différentes valeurs de $C$.

De toutes ces solutions, nous déterminons le coefficient $C^{*}$ vérifiant : Erreur $\left(C^{*}\right)=$ minimum $($ Erreur $(C))$ grâce au critère $(26)$. Le paramètre de diffusion retenu est $C^{*}=21,4$ avec Erreur $\left(C^{*}\right)=0,29 \%$ (Fig. 14). Sur la figure 15 , on présente la variation de l'erreur en fonction du paramètre de diffusion où l'on voit clairement l'optimum qui correspond à une portion assez plate de la courbe. Il existe donc une plage assez large de valeur de $C$ qui donne un résultat tout à fait satisfaisant.

Pour chaque rapport $\frac{R_{\mathrm{racc}}}{R_{\mathrm{b}}}$ donné, nous déterminons cette valeur $C^{*}$. Sur la figure 16 , on peut observer une large plage $[0,1 ; 0,8]$ pour laquelle le paramètre $C^{*}$ dépend très peu du rapport $\frac{R_{1}}{R_{2}}$. Il semble donc qu'une valeur unique de $C^{*}$ convienne de manière très satisfaisante (erreur $<1 \%$ ) à toutes les géométries de raccordement. Ce résultat n'est valable que pour une géométrie variant peu autour des dimensions de contact galet-cylindre. Dans la mise en place d'un outil de simulation d'usure, c'est bien le cas : l'usure génère des variations de dimensions assez faibles.

Après diffusion, les résultats de l'approche semihertzienne sont très analogues à ceux obtenus par l'approche exacte : les « pointes » sont fortement atténuées et les dimensions de la zone de contact correspondent mieux à la solution exacte (Fig. 17).

Nous chiffrons dans (Tab. 2) les dimensions de la zone de contact du galet en biais par l'approche semihertzienne et l'approche exacte avec et sans diffusion. Il est clair que le modèle semi-hertzien donne de très bonnes approximations. Nous désignons par $L_{x}$ la largeur de la zone de contact et par $L_{y}$ sa longueur.

\section{Conclusions}

Nous avons présenté quelques outils de résolution approchée du problème de contact. Une étude comparative de l'approche simplifiée FastSim avec le modèle exact nous permet de retenir le choix d'une répartition elliptique de la pression de contact. Les écarts constatés par rapport à l'approche exacte sur la puissance surfacique et la puissance linéique, paramètres déterminants dans la simulation d'une loi d'usure, sont plus élevés en répartition parabolique qu'en répartition elliptique. Le problème de la vitesse de glissement qui tend vers l'infini sur le bord de 


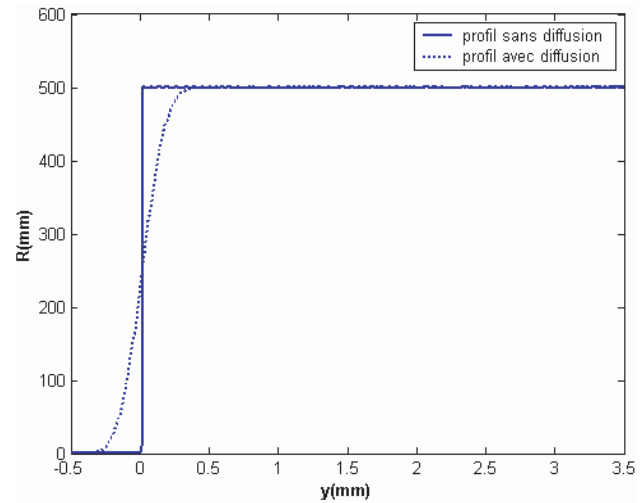

(a)

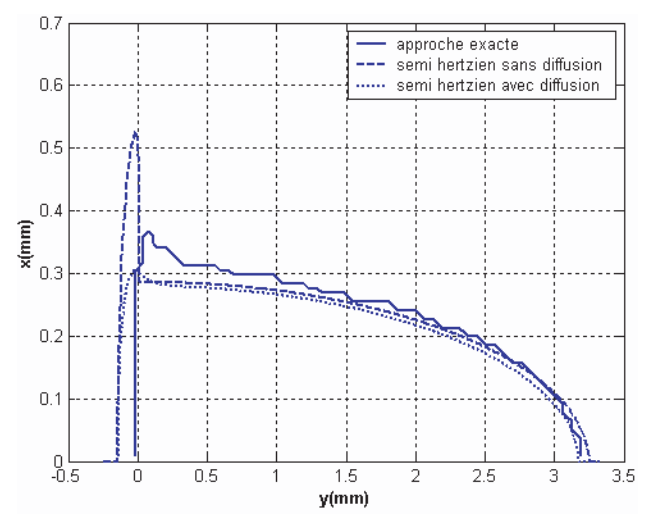

(b)

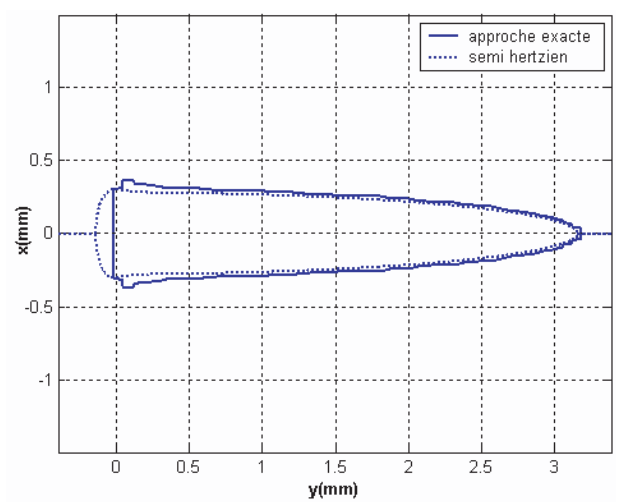

(c)

Fig. 14. Résultat de la diffusion avec le paramètre optimal $C^{*}$ : (a) variations des courbures du galet dans le plan $(y O z)$ avant et après diffusion, (b) forme de la zone de contact : confrontation des trois approches, (c) bonne approximation de la méthode semi-hertzienne avec diffusion.

fuite, qui semble être l'argument principal pour le choix de la répartition parabolique donne une puissance dissipée surfacique finie pour une répartition elliptique.

Dans le cas des courbures non constantes, une approche semi-hertzienne issue des travaux antérieurs de $[4,5,14]$ a été mise en place. Elle nécessite une correction de la courbure mais aussi une procédure de diffusion

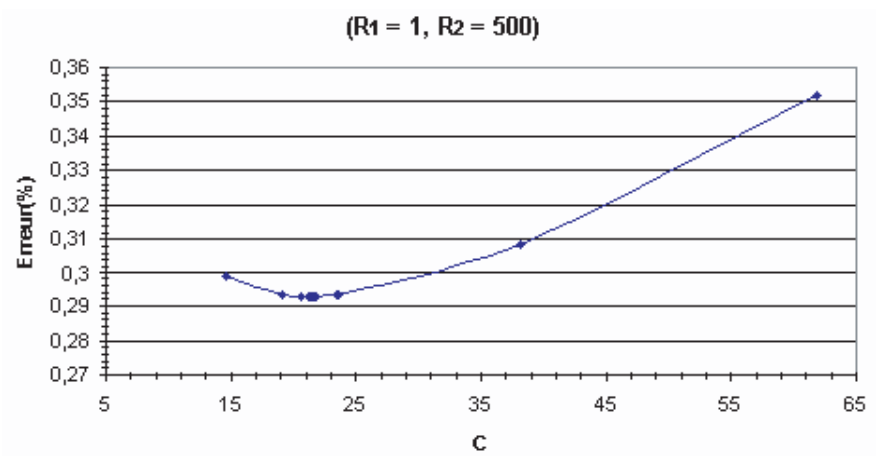

Fig. 15. Variation de l'erreur en fonction de $C$ dans le cas $R_{2}=500 \mathrm{~mm}$ et $R_{1}=1 \mathrm{~mm}$ : la fonction erreur est convexe et le minimum est atteint au point de coordonnées $(21,42$; $0,29)$.

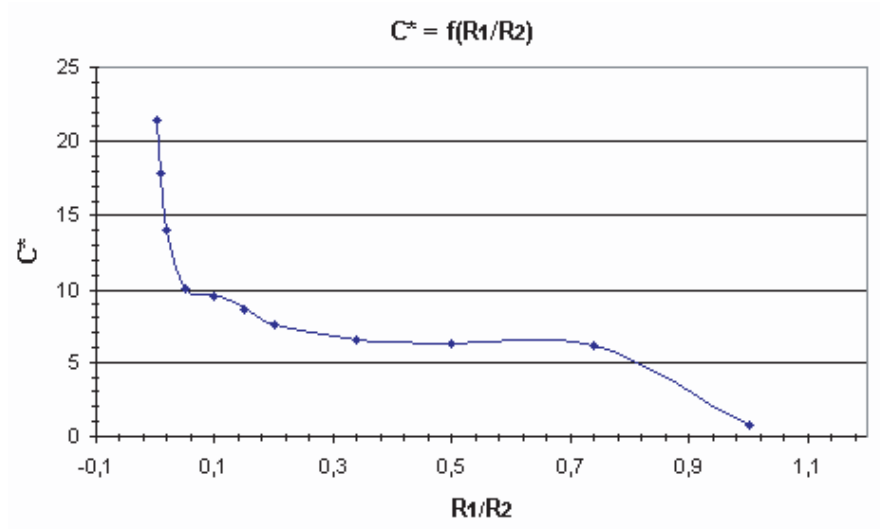

Fig. 16. Évolution du paramètre de diffusion pour des rayons de raccordement quelconques allant de 1 à $100 \mathrm{~mm}$ dans le cas où $R_{2}=500 \mathrm{~mm}$.

Tableau 2. Comparaison des dimensions de la zone de contact par l'approche exacte et le modèle semi-hertzien.

\begin{tabular}{|c|c|c|c|c|c|}
\cline { 3 - 6 } \multicolumn{2}{c|}{} & \multicolumn{5}{|c|}{ Rayons de raccordement } \\
\cline { 3 - 6 } \multicolumn{2}{c|}{} & 1 & 10 & 50 & 100 \\
\hline \multirow{2}{*}{ Semi-hertzien } & $L_{x}(\mathrm{~mm})$ & 0,61 & 0,6 & 0,56 & 0,53 \\
& $L_{y}(\mathrm{~mm})$ & 3,32 & 3,5 & 3,8 & 4,1 \\
\hline Approche & $L_{x}(\mathrm{~mm})$ & 0,7 & 0,7 & 0,61 & 0,55 \\
exacte & $L_{y}(\mathrm{~mm})$ & 3,21 & 3,38 & 3,7 & 4,1 \\
\hline \multicolumn{2}{|c|}{ Erreur en $L_{x}(\%)$} & 13 & 14 & 8 & 4 \\
\hline \multicolumn{2}{|c|}{ Erreur en $L_{y}(\%)$} & 3 & 4 & 3 & 0 \\
\hline
\end{tabular}

permettant d'estomper les irrégularités de la zone de contact dues à la transition brutale des courbures pour bomber $\mathrm{du}$ 《 galet en biais » au rayon de raccordement. Cette méthode permet d'obtenir une bonne corrélation avec l'approche exacte sans introduire plus d'un seul coefficient $C^{*}$ de réglage de la méthode. En effet, une optimisation de la méthode des éléments diffus montre que la même valeur $C^{*}$ convient à une large plage de rapport $R_{1} / R_{2}$ ce qui rend l'approche assez générale.

Cette approche est actuellement testée sur des géométries assez critiques : conformité des contacts, existence de zone à courbure négative, influence du rapport des courbures $B / A$. Parallèlement, la 

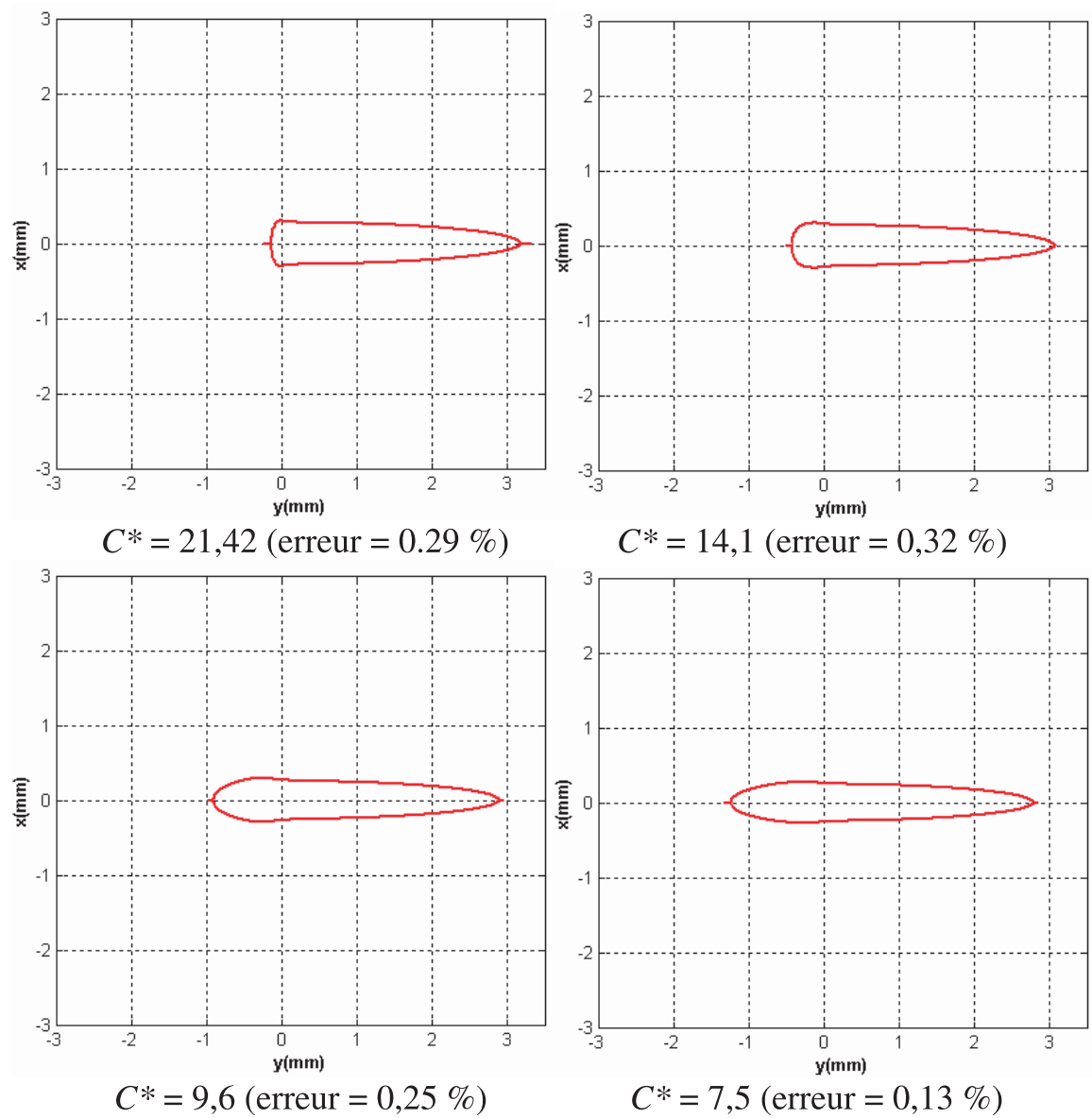

Fig. 17. Formes de la zone de contact par la méthode semi hertzienne avec diffusion pour des rayons de raccordement valant respectivement $1,10,50$ et $100 \mathrm{~mm}$.

simulation numérique de l'usure de manière itérative avec réactualisation des profils au cours du temps est en cours couplée à l'approche probabiliste pour la prise en compte des dispersions expérimentales dans la démarche.

\section{Références}

[1] L. Chevalier, H. Chollet, Endommagement des pistes de roulement, Mec. Ind. 1 (2000) 77-103.

[2] L. Chevalier, S. Cloupet, C. Soize, Probabilistic approach for wear modelling in steady state rolling contact, accepted for publication in Wear (November 2004)

[3] J.J. Kalker, A Fast Algorithm for the Simplified Theory of Rolling Contact, Vehicle Syst. Dyn. 11 (1982) 1-13

[4] J.B. Ayasse, H. Chollet, Determination of the Wheel Rail Contact Patch in Semi-Hertzian Conditions, Vehicle Syst. Dyn., in press

[5] J.B. Ayasse, H. Chollet, Contact Semi-Hertzien, Rapport, LTN-INRETS, 2002

[6] J.F. Archard, Contact and rubbing of flat surfaces, J. Appl. Phys. 24 (1953) 981-988

[7] K.L. Johnson, Contact Mechanics, Cambridge University Press, 1985

[8] J.J. Kalker, On the rolling contact of two elastic bodies in presence of dry friction, Thesis, Dpt of Mechanical Engineering, Delft University of Technology, 1967, p. 155
[9] A.E.H. Love, A Treatise on the Mathematical Theory of Elasticity, Dover, New York, 1944

[10] J. Boussinesq, Application des potentiels à l'équilibre et du mouvement des solides élastiques, Gauthier-Villars, Paris, 1885

[11] H. Hertz, Über die berührung fester elasticher körper (on the contact of elastic solids), J. Reine Angew. Math. 92 (1882) 156-171, translated and reprinted in Hertz's Miscelaneous Papers, MacMillan \& Co, London, 1896

[12] J.J. Kalker, B. Jacobson, Rolling Contact Phenomena : Linear Elasticity, Berlin, 2000

[13] B. Soua, Étude de l'usure et de l'endommagement du roulement ferroviaire avec essieux non rigides, Thèse ENPC, 1997

[14] W. Kik, J. Piotrowski, A fast approximative method to calculate normal load at contact between wheel and rail, and creep forces during rolling, Warsaw Technical University, 2nd mini-conference on contact mechanics and wear of rail/wheel systems, Budapest, July 29-31, 1996

[15] T. Telliskivi, Simulation of wear in a rolling-sliding contact by a semi-Winkler model and the Archard's wear law, Wear, 2003

[16] M.C. Costa, J. Coulomb, Y. Maréchal, S.I. Nabeta, An Adaptative Method Applied to the Diffuse Element Approximation in Optimization Process, IEEE T. Magn. 37 (2001) 3418-3422.

[17] B. Nayroles, G. Touzot, P. Villon, La méthode des éléments diffus, C. R. Acad. Sci. 